\title{
Submanifolds in Poisson geometry: a survey
}

Marco Zambon

\begin{abstract}
We describe various classes of submanifolds of a Poisson manifold $M$, both in terms of tensors on $M$ and of constraints: coisotropic submanifolds, PoissonDirac submanifolds (which inherit a Poisson structure), and the very general class of pre-Poisson submanifolds. We discuss embedding results for these classes of submanifolds, quotient Poisson algebras associated to them, and their relationship to subgroupoids of the symplectic groupoid of $M$.
\end{abstract}

\section{Poisson geometry}

The phase space of a physical system, in the hamiltonian formalism, is usually given the structure of a symplectic manifold. When the system is invariant under symmetries, it makes sense to consider the "reduced" phase space obtained quotienting the original phase space by the symmetries. The reduced phase space in general is no longer symplectic, but rather has the structure of a Poisson manifold. We recall some basic facts about Poisson manifolds (see Weinstein's seminal 1983 paper [14] or the book [12] for detailed expositions).

The algebraic definition of Poisson manifold is the following:

Definition 1. A Poisson manifold is a manifold $M$ such that the algebra of functions $C^{\infty}(M)$ is endowed with a Lie bracket $\{\cdot, \cdot\}$ satisfying $\{f, g h\}=\{f, g\} h+g\{f, h\}$ for all $f, g, h$.

Often it is convenient to use a more geometric definition:

Definition 2. A Poisson manifold is a manifold $M$ endowed with a bivector field $\pi \in \Gamma\left(\wedge^{2} T M\right)$ satisfying $[\pi, \pi]=0$.

Marco Zambon

Universidade do Porto, Departamentos de Matematica Pura, Rua do Campo Alegre, 687, 4169-007

Porto, Portugal, e-mail: mzambon@fc.up.pt 
Here $[\cdot, \cdot]$ denotes the Schouten bracket of multivector fields, which extends the Lie bracket of vector fields on $M$. The Poisson bracket $\{\cdot, \cdot\}$ and $\pi$ are related by $\{f, g\}=\pi(d f, d g)$.

Let $M$ be a Poisson manifold. The bivector field $\pi \in \Gamma\left(\wedge^{2} T M\right)$ can be equivalentely described by

$$
\sharp: T^{*} M \rightarrow T M, \sharp \xi=\pi(\xi, \cdot),
$$

a bundle map which is skew-symmetric (i.e., $\left.\sharp^{*}=-\sharp\right)$. One can show that $\operatorname{Im}(\sharp) \subset$ $T M$ is an involutive singular distribution ${ }^{1}$, so $M$ is foliated by leaves (immersed submanifolds of varying dimensions) whose tangent spaces are exactly given by $\operatorname{Im}(\sharp)$.

At every $p \in M$ the kernel of $\sharp_{p}: T_{p}^{*} M \rightarrow \operatorname{Im}\left(\pi_{p}\right)$ is the annihilator $\left(\operatorname{Im}\left(\sharp_{p}\right)\right)^{\circ}:=$ $\left\{\xi \in T_{p}^{*} M:\left.\xi\right|_{\operatorname{Im}\left(\sharp_{p}\right)}=0\right\}$, hence inverting the induced isomorphism

$$
T_{p}^{*} M /\left(\operatorname{Im}\left(\sharp_{p}\right)\right)^{\circ} \cong \operatorname{Im}\left(\sharp_{p}\right)^{*} \rightarrow \operatorname{Im}\left(\sharp_{p}\right)
$$

we obtain a linear symplectic from $\omega_{p}$ on $\operatorname{Im}\left(\sharp_{p}\right)$. One can show that the 2-form $\omega$ on each leaf $\mathscr{O}$ is actually symplectic. So we conclude that an equivalent characterization of Poisson manifold is the following: a manifold foliated by leaves of varying dimensions, each of which carries a symplectic form varying smoothly with the leaf.

Example 1. a) A symplectic form $\omega$ on a manifold $M$ can be regarded as a Poisson bivector field, by the requirement $\sharp=-\tilde{\omega}^{-1}$ where $\tilde{\omega}: T M \rightarrow T^{*} M, v \mapsto \omega(v, \cdot)$.

b) If $\mathfrak{g}$ is a finite dimensional real Lie algebra, then $\mathfrak{g}^{*}$ has a natural Poisson structure, determined by $\{v, w\}=[v, w]$ where $v, w \in \mathfrak{g}$ are also viewed as linear functions on $\mathfrak{g}^{*}$. The symplectic leaves of $\mathfrak{g}^{*}$ are the coadjoint orbits.

For instance, the symplectic leaves in $\mathfrak{s u}(2)^{*}$ are spheres centered at the origin, with symplectic form growing linearly with the radius. The Poisson bivector field is given by $x_{3} \frac{\partial}{\partial x_{1}} \wedge \frac{\partial}{\partial x_{2}}+x_{1} \frac{\partial}{\partial x_{2}} \wedge \frac{\partial}{\partial x_{3}}+x_{2} \frac{\partial}{\partial x_{3}} \wedge \frac{\partial}{\partial x_{1}}$ in suitable linear coordinates on $\mathfrak{s u}(2)^{*}$.

c) On every manifold $M$, setting $\pi=0$ one obtains a Poisson bivector field. Each point of $M$ is a symplectic leaf.

\subsection{Submanifolds and symplectic leaves}

Let $(M, \pi)$ be a Poisson manifold. In this Subsection we use the symplectic foliation described above as a guide to determine classes of submanifolds.

A natural class of submanifolds are symplectic leaves (leaves $\mathscr{O}$ endowed with the symplectic form $\omega$ as above). Generalizing this slightly, we obtain Poisson submanifolds, which are just unions of (open subsets of) symplectic leaves.

Definition 3. [12, §6.6] $N \subset(M, \pi)$ is a Poisson submanifold iff $\pi_{p} \in \wedge^{2} T_{p} N$ for every $p \in N$.

\footnotetext{
${ }^{1}$ Indeed $T^{*} M$ is a Lie algebroid (see $\S 1.2$ ), and the image of the anchor of any Lie algebroid is an involutive singular distribution.
} 
Equivalent conditions are $\sharp T N^{\circ}=\{0\}$ or $\operatorname{Im}\left(\left.\sharp\right|_{N}\right) \subset T N$.

Given a symplectic vector space $(V, \omega)$, an interesting class of subspaces $W$ are the coisotropic ones, i.e. those for which $W^{\omega} \subset W$. Another interesting class is given by the symplectic subspaces, i.e. those for which $W^{\omega} \cap W=\{0\}$.

It is natural to consider submanifolds of the Poisson manifold $(M, \pi)$ whose intersections with the symplectic leaves are coisotropic or symplectic submanifolds of the leaves. Since these intersections are usually not smooth, we are lead to consider tangent spaces.

Lemma 1. Let $N$ be a submanifold of $(M, \pi)$. For all $p \in N$ denote by $(\mathscr{O}, \omega)$ the symplectic leaf through $p$. The symplectic orthogonal of $T_{p} N \cap T_{p} \mathscr{O}$ in $\left(T_{p} \mathscr{O}, \omega_{p}\right)$ is $\left(T_{p} N \cap T_{p} \mathscr{O}\right)^{\omega_{p}}=\sharp T_{p} N^{\circ}$. Hence:

- $T_{p} N \cap T_{p} \mathscr{O}$ is a coisotropic subspace of $\left(T_{p} \mathscr{O}, \omega_{p}\right) \Leftrightarrow \sharp T_{p} N^{\circ} \subset T_{p} N$,

- $T_{p} N \cap T_{p} \mathscr{O}$ is a symplectic subspace of $\left(T_{p} \mathscr{O}, \omega_{p}\right) \Leftrightarrow \sharp T_{p} N^{\circ} \cap T_{p} N=\{0\}$.

The above lemma follows from a simple computation and from $\left(T_{p} N \cap T_{p} \mathscr{O}\right) \cap$ $\left(T_{p} N \cap T_{p} \mathscr{O}\right)^{\omega_{p}}=\sharp T_{p} N^{\circ} \cap T_{p} N$.

Submanifolds satisfying the first condition above are called coisotropic. In some cases they are the replacement in Poisson geometry of the symplectic-geometric notion of "Lagrangian", see Ex. 2 b). Those that satisfy the second condition and an additional smoothness requirement are called Poisson-Dirac submanifolds. We will elaborate on them in $\S 2$ and $\S 3$ respectively. The intersection between the classes of coisotropic and Poisson-Dirac submanifolds are exactly the Poisson submanifolds.

\subsection{Lie algebroids and Dirac manifolds}

In order to determine further classes of submanifolds of a Poisson manifold, we introduce two notions that are canonically associated to Poisson geometry.

A Lie algebroid $[12, \S 16]$ consists of a vector bundle $A \rightarrow N$ together with a Lie bracket on the space of sections $\Gamma(A)$ and a bundle map $\rho: A \rightarrow T N$ (called anchor) satisfying $[a, f \cdot b]=\rho(a) f \cdot b+f \cdot[a, b]$ for all sections $a, b$ and functions $f$. When $N$ is a point, this notion reduces to that of Lie algebra.

For any Poisson manifold $(M, \pi), T^{*} M$ is naturally a Lie algebroid $[12, \S 13]$ with anchor $-\sharp: T^{*} M \rightarrow T M$, and bracket determined by $[d f, d g]:=d\{f, g\}$.

A Dirac manifold $[9, \S 2.2]$ is a manifold $P$ together with a subbundle $L \subset T P \oplus$ $T^{*} P$ which is maximal isotropic w.r.t. the pairing $\left\langle X_{1} \oplus \xi_{1}, X_{2} \oplus \xi_{2}\right\rangle=\frac{1}{2}\left(i_{X_{2}} \xi_{1}+\right.$ $\left.i_{X_{1}} \xi_{2}\right)$ and whose sections are closed under the Courant bracket

$$
\left[X_{1} \oplus \xi_{1}, X_{2} \oplus \xi_{2}\right]=\left(\left[X_{1}, X_{2}\right] \oplus \mathscr{L}_{X_{1}} \xi_{2}-\mathscr{L}_{X_{2}} \xi_{1}+\frac{1}{2} d\left(i_{X_{2}} \xi_{1}-i_{X_{1}} \xi_{2}\right)\right) .
$$

on $\Gamma\left(T P \oplus T^{*} P\right)$. Given any submanifold $N \subset(P, L)$, one can pull back the Dirac structure $L$ to $N$, by defining $L_{N}=L \cap\left(\left.T N \oplus T^{*} M\right|_{N}\right) / L \cap T N^{\circ}$. This subset of 
$T N \oplus T^{*} N$ is not necessarily a smooth subbundle, but when it is, it is automatically a Dirac structure on $N[9, \S 3.1]$.

For any Poisson manifold $(M, \pi), L:=\operatorname{graph}(\pi):=\left\{(\sharp \xi, \xi): \xi \in T^{*} M\right\}$ is a Dirac structure. Viewing a Poisson manifold as a Dirac manifold has the advantage that, even though we can not restrict the Poisson bivector field $\pi$ to a submanifold $N \subset M$ (except when $N$ is a Poisson submanifold), $N$ is always endowed with the geometric structure $L_{N}$, which is a Dirac structure whenever $L_{N}$ is a smooth subbundle.

\section{Coisotropic submanifolds}

In this section we elaborate on coisotropic submanifolds [12, §6.4].

Definition 4. $N \subset(M, \pi)$ is a coisotropic submanifold iff $\sharp T N^{\circ} \subset T N$.

Let $N$ be a submanifold of $(M, \pi)$. Then

$$
\mathscr{I}:=\left\{f \in C^{\infty}(M):\left.f\right|_{N}=0\right\}
$$

is a multiplicative ideal of the Poisson algebra $C^{\infty}(M)$. The submanifolds for which $\mathscr{I}$ is also a Poisson subalgebra (i.e., $\{\mathscr{I}, \mathscr{I}\} \subset \mathscr{I}$ ) are exactly the coisotropic submanifolds. Those satisfying the stronger condition that $\mathscr{I}$ is a Poisson ideal (i.e., $\left.\left\{\mathscr{I}, C^{\infty}(M)\right\} \subset \mathscr{I}\right)$ are exactly the Poisson submanifolds.

In the physics literature, sometimes submanifolds are specified by constraints, i.e. open subsets $\left\{U_{\alpha}\right\}$ of $M$ and, for each $\alpha$, independent functions $\varphi_{\alpha}^{1}, \ldots, \varphi_{\alpha}^{k}$ defined on $U_{\alpha}$ such that $N \cap U_{\alpha}$ is the common zero set of $\varphi_{\alpha}^{1}, \ldots, \varphi_{\alpha}^{k}$. As we just saw, the coisotropic submanifolds are exactly those given by so-called first class constraints, i.e. constraints satisfying $\left.\left\{\varphi^{i}, \varphi^{j}\right\}\right|_{N}=0$.

Example 2. a) Poisson submanifolds are coisotropic.

b) If $\varphi:\left(M_{1}, \pi_{1}\right) \rightarrow\left(M_{2}, \pi_{2}\right)$ is a Poisson morphism, then its graph is coisotropic in $\left(M_{1} \times M_{2}, \pi_{1}-\pi_{2}\right)$.

c) If $\mathfrak{h}$ is a Lie subalgebra of the Lie algebra $\mathfrak{g}$, then $\mathfrak{h}^{\circ}$ is a coisotropic submanifold of $\mathfrak{g}^{*}$. (See Ex. 5 for an extension of this example.)

Remark 1. The intersection of a coisotropic submanifold with the symplectic leaves $\mathscr{O}$ of $M$ is usually not clean: for instance, the symplectic foliation of $\mathfrak{s u}(2)^{*}$ is given by concentric spheres in $\mathbb{R}^{3}$. Any plane $N$ in $\mathfrak{s u}(2)^{*}$ not containing the origin is coisotropic, has a (unique) point $p$ at which $N$ is tangent to a symplectic sphere $\mathscr{O}$, and at that point $T_{p}(N \cap \mathscr{O}) \neq T_{p} N \cap T_{p} \mathscr{O}$.

Coisotropic submanifolds enjoy nice properties: their conormal bundle $T N^{\circ}$ is a Lie subalgebroid of $T^{*} M$, and they admit a natural quotient which is again a Poisson manifold, provided it is smooth. We will discuss these properties for the more general class of pre-Poisson submanifolds in $\S 4.3$ and $\S 4.2$ respectively. 


\section{Poisson-Dirac submanifolds}

Poisson-Dirac submanifolds, introduced by Crainic and Fernandes $[10, \S 8]$ in 2002, are the submanifolds of $(M, \pi)$ which have a canonically induced Poisson structure.

Definition 5. [10, Def. 4, $\S 8$ ]. $N$ is a Poisson-Dirac submanifold of $(M, \pi)$ if it has a Poisson structure such that:

(i) $N$ intersects cleanly ${ }^{2}$ the symplectic leaves of $M$, and the symplectic leaves of $N$ are the connected components of $N \cap \mathscr{O}$ as $\mathscr{O}$ ranges over all symplectic leaves of $M$,

(ii) $N \cap \mathscr{O}$ is a symplectic submanifold of $\mathscr{O}$, for every symplectic leaf $\mathscr{O}$ of $M$.

An alternative characterization, along the lines of our reasoning at the end of $\S 1$, is the following. Let $N$ be a submanifold of $M$ such that, for any $p \in N, T_{p} N \cap T_{p} \mathscr{O}$ is a symplectic subspace of $\left(T_{p} \mathscr{O}, \omega_{p}\right)$. Here $(\mathscr{O}, \omega)$ denotes the symplectic leaf through $p$. Then the restriction of $\omega_{p}$ to $T_{p} N \cap T_{p} \mathscr{O}$ is a non-degenerate bilinear form, and inverting it we obtain a bivector $\left(\pi_{N}\right)_{p} \in \wedge^{2} T_{p} N$. Notice that in general $\pi_{N}$ is not a smooth section of $\wedge^{2} T N$ (see [10, Ex. 3, §8]).

Definition 6. [10, Cor. 11, $\S 8] N \subset(M, \pi)$ is a Poisson-Dirac submanifold iff $\sharp T N^{\circ} \cap T N=\{0\}$ and the induced tensor $\pi_{N}$ on $N$ is smooth.

In that case, $\pi_{N}$ is automatically a Poisson tensor [10, Prop. $\left.6, \S 8\right]$. The name "Poisson-Dirac" derives from the fact that $\operatorname{graph}\left(\pi_{N}\right)$ is equal to $L_{N}$, the Dirac structure obtained pulling back $\operatorname{graph}(\pi)$ via the inclusion $N \hookrightarrow M$.

Any submanifold $N$ such that $\sharp T N^{\circ} \cap T N=\{0\}$ and for which $\sharp T N^{\circ}$ has constant rank, is automatically Poisson-Dirac ${ }^{3}$. Indeed the latter condition implies that pulling back the Dirac structure $\operatorname{graph}(\pi)$ we obtain a smooth subbundle of $T N \oplus T^{*} N$ (see $\S 1.2$ ), which hence is the graph of a smooth bivector field on $N$. In this case the Poisson bracket on $N$ is computed as follows:

$$
\{f, g\}:=\left.\{\hat{f}, \hat{g}\}\right|_{N}
$$

where $\hat{f}, \hat{g} \in C^{\infty}(M)$ are extensions of $f, g \in C^{\infty}(N)$ such that $\left.d f\right|_{\sharp T N^{\circ}}=0$.

Examples of Poisson-Dirac submanifolds are:

Example 3. a) If $(M, \omega)$ is a symplectic manifold, then a submanifold $N$ is PoissonDirac iff it is a symplectic submanifold.

b) Poisson submanifolds.

c) Lie-Dirac submanifolds, in particular cosymplectic submanifolds. We will elaborate on them in $\S 3.1$ and $\S 3.2$.

\footnotetext{
${ }^{2}$ This means that $N \cap \mathscr{O}$ is a manifold with $T(N \cap \mathscr{O})=T N \cap T \mathscr{O}$.

${ }^{3}$ It also falls into the more restrictive class of quasi-Dirac submanifolds [13, Def. 2.2], see also [10, Prop. 7, §8].
} 
Within the class of Poisson-Dirac submanifolds, the cosymplectic ones and the Poisson submanifolds lie at opposite extremes: for the former the rank of $\sharp T N^{\circ}$ is maximized, for the latter it is zero.

\subsection{Lie-Dirac submanifolds}

Lie-Dirac submanifolds were introduced by $\mathrm{Xu}^{4}$ in 2001 [15]. They are special cases of Poisson-Dirac submanifolds [10, §8.3].

Definition 7. [15, Def. 2.1] $N \subset(M, \pi)$ is a Lie-Dirac submanifold iff there exists a subbundle $E$ with $\left.T M\right|_{N}=T N \oplus E$ such that $E^{\circ}$ is a Lie subalgebroid of $T^{*} M$.

Recall [11, Def. 4.3.14] that if $A \rightarrow M$ is a Lie algebroid with anchor $\rho$, a subbundle $B \rightarrow N$ is a Lie subalgebroid if $\rho(B) \subset T N$ and for all sections $X, Y$ of $A$ one has $\left(\left.X\right|_{N},\left.\left.Y\right|_{N} \in \Gamma(B) \Rightarrow[X, Y]\right|_{N} \in \Gamma(B)\right)$ and $\left(\left.X\right|_{N}=0,\left.\left.Y\right|_{N} \in \Gamma(B) \Rightarrow[X, Y]\right|_{N}=0\right)$.

The embedding $T^{*} N \rightarrow T^{*} M$, given by the canoncial identification between the vector bundles $T^{*} N$ and $E^{\circ}$, is actually a morphism of Lie algebroids, giving rise to a Lie subalgebroid of $T^{*} M$ [15, Thm. 2.3 iii)]. (Here the Lie algebroid structures or $T^{*} N$ and $T^{*} M$ are those given by the Poisson bivector fields on $N$ and $M$.) The fact that Lie-Poisson submanifolds come with a canonical Lie subalgebroid of $T^{*} M$ accounts for several good properties of Lie-Poisson submanifolds, see for example Prop. 4.

A characterization in terms of functions is

Definition 8. [13, Def. 2.1] $N \subset(M, \pi)$ is a Lie-Dirac submanifold iff there exists a subbundle $E$ containing $\sharp T N^{\circ}$ for which $\left.T M\right|_{N}=T N \oplus E$, such that for all $f, g \in$ $C^{\infty}(M)$

$$
\left.d f\right|_{E}=0,\left.d g\right|_{E}=\left.0 \Rightarrow d\{f, g\}\right|_{E}=0 .
$$

Being a Lie-Dirac submanifold is global property of the submanifold $N$ : if we can find subbundles as above on open subsets of $N$, in general we can not glue them into a subbundle $E$ over $N$ as above.

Example 4. a) Points of Poisson manifolds are Lie-Dirac submanifolds.

b) Cosymplectic submanifolds, which we will introduce in $\S 3.2$, are Lie-Dirac submanifolds [15, Cor 2.11].

c) Symplectic leaves of Poisson manifolds are usually not Lie-Dirac submanifolds. For instance, the symplectic foliation of $\mathfrak{s u}(2)^{*}$ consist of concentric spheres, and among these only the origin is a Lie-Dirac submanifold. The exact obstruction for regular ${ }^{5}$ symplectic leaves is given in [10, Cor 13, §8], see also [15, Ex. 2.17].

${ }^{4} \mathrm{Xu}$ introduced them with the name "Dirac submanifolds"; the name "Lie-Dirac" was proposed in [10].

${ }^{5}$ I.e., leaves such that all the symplectic leaves in a neighborhood have the same dimension. 


\subsection{Cosymplectic submanifolds}

The notion of cosymplectic submanifold is much older than that of Poisson-Dirac or Lie-Dirac submanifold.

Definition 9. $[14, \S 1] N \subset(M, \pi)$ is a cosymplectic submanifold iff $\sharp T N^{\circ} \oplus T N=$ $\left.T M\right|_{N}$.

Hence cosymplectic submanifolds are exactly the submanifolds given by second class constraints, i.e. constraints $\left\{\varphi^{A}\right\}$ such that $\left.\left\{\varphi^{A}, \varphi^{B}\right\}\right|_{p}$ is an invertible matrix at all points $p \in N$. This follows from the fact for any Poisson-Dirac submanifold $\sharp T N^{\circ}$ is a symplectic subbundle, see Lemma 1 .

Cosymplectic submanifolds constitute a useful tool in hamiltonian mechanics. Let $(M, \pi)$ be the Poisson manifold representing the phase space of a physical system. Sometimes the physical system is constrained to a submanifold $N \subset M$ with an induced Poisson structure (a Poisson-Dirac submanifold), and one would like to express the induced Poisson bracket $\{\cdot, \cdot\}_{N}$ on $N$ in terms of the Poisson bracket $\{\cdot, \cdot\}$ on $M$.

The case when $N$ is cosymplectic is well-known in the physics literature, and has been threated using the so-called Dirac bracket. We describe it as follows. Let $\varphi^{1}, \ldots, \varphi^{k}$ be constraints for the cosympectic submanifold $N$ defined on an open subset $U \subset M$. Since the matrix $C^{A B}:=\left\{\varphi^{A}, \varphi^{B}\right\}$ is invertible on $N \cap U$, we may assume that it is invertible on $U$, shrinking $U$ if necessary. We denote its inverse by $C_{A B}$. The Dirac bracket is the bracket on $C^{\infty}(U)$ defined by

$$
\{f, g\}_{\text {Dirac }}:=\{f, g\}-\left\{f, \varphi^{A}\right\} C_{A B}\left\{\varphi^{B}, g\right\} .
$$

It is a Poisson bracket, and it allows to recover easily the bracket $\{\cdot, \cdot\}_{N}$ on $N$ : the latter is computed extending in any arbitrary way functions on $N$ to functions on $M$ and taking their Dirac bracket. (Notice that computing $\{\cdot, \cdot\}_{N}$ by means of the Poisson bracket $\{\cdot, \cdot\}$ on $M$, as in $\S 3$, requires specific extensions of the functions on $N$ : the extensions must annihilate $\sharp T N^{\circ}$.)

We explain the above statement as follows. Denote by $\pi_{\text {Dirac }}$ the Poisson bivector field $U$ given by the Dirac bracket. It can be shown $[4, \S 5.1]$ that the level sets of the constraints (in particular $N$ ) are cosymplectic submanifolds of $(M, \pi)$ and also Poisson submanifolds of $\left(U, \pi_{\text {Dirac }}\right)$, and that the Poisson structures on the level sets induced by $\pi$ and $\pi_{\text {Dirac }}$ coincide.

\section{Pre-Poisson submanifolds}

Given a symplectic manifold $(X, \Omega)$, a submanifold $\imath: C \hookrightarrow X$ is called presymplectic if the characteristic distribution $\operatorname{ker}\left(\imath^{*} \Omega\right)=T C \cap T C^{\Omega}$ has constant rank along $C$, or equivalently if $T C+T C^{\Omega}$ has constant rank. In this Section we consider an 
extension of the notion of presymplectic submanifold to Poisson geometry, and in the three Subsections we establish various interesting properties.

Let $(M, \pi)$ be a Poisson manifold and $N$ a submanifold. It is natural to consider the kernel of $\left.\omega\right|_{T_{p} N \cap T_{p} \mathscr{O}}$, where $\omega$ is the symplectic form on the symplectic leaf $\mathscr{O}$ through $p$, and impose that it have constant rank for all $p \in N$. By Lemma 1 this amounts to asking that $\operatorname{char}(N):=T N \cap \sharp T N^{\circ}$ has constant rank along $N$. This turns out not to be a good notion. For instance, $\left.\operatorname{char}(N) \subset T M\right|_{N}$ may have constant rank but fail to be a smooth subbundle of $\left.T M\right|_{N}$ (see Ex. 5.7 of [7]).

Instead of the intersection of $T N$ and $\sharp T N^{\circ}$, it is better to consider their sum:

Definition 10. [7, Def. 2.2] A submanifold $N$ of a Poisson manifold $(M, \pi)$ is called pre-Poisson if the rank of $T N+\sharp T N^{\circ}$ is constant along $N$.

Such submanifolds were first considered by Calvo and Falceto [2, 3] in 2004, and studied by Cattaneo and the author in [6],[7]. A first good property of $T N+\sharp T N^{\circ}$ is the following: if the rank of $\sharp T N^{\circ}+T N$ is constant, then it is automatically a smooth subbundle of $\left.T M\right|_{N}$, because smooth sections spanning $\sharp T N^{\circ}+T N$ can be easily constructed from a smooth frame for $T N$ and the image under $\left.\sharp\right|_{N}$ of a smooth frame for $T N^{\circ}$.

Example 5. 1) If $(M, \omega)$ is a symplectic manifold, a submanifold $N$ is pre-Poisson iff it is presymplectic.

2) Coisotropic submanifolds (see $\S 2$ ) are pre-Poisson.

3) Poisson-Dirac submanifolds (see $\S 3$ ) or even Lie-Dirac submanifolds (see $\S 3.1$ ) are usually not pre-Poisson, but cosymplectic submanifolds (see $\S 3.2$ ) are.

4) Let $\mathfrak{h}$ be a Lie subalgebra of a Lie algebra $\mathfrak{g}$ and fix $\lambda \in \mathfrak{g}^{*}$. Then the affine subspace $\mathfrak{h}^{\circ}+\lambda$ is pre-Poisson $[6, \S 5]$.

To put into perspective Def. 10 , let $N$ be an arbitrary submanifold of $(M, \pi)$ and consider three "singular subbundles":

- $\sharp T N^{\circ}$

- $\operatorname{char}(N)=T N \cap \sharp T N^{\circ}$

- $T N+\sharp T N^{\circ}$.

The first two are the domain and kernel respectively of

$$
\phi: \sharp T N^{\circ} \rightarrow v N,
$$

the restriction of the projection $p r_{v N}:\left.T M\right|_{N} \rightarrow v N:=\left.T M\right|_{N} / T N$. The image of $\phi$ is $p r_{v N}\left(\sharp T N^{\circ}+T N\right)$. Hence it is clear that

Lemma 2. Let $N$ be a submanifold of $M$. Whenever any two of char $(N), \sharp T N^{\circ}, \sharp T N^{\circ}+$ $T N$ have constant rank, then the remaining one also does.

We elaborate on the properties that $N$ has when one of the three above "singular subbundles" has constant rank. By definition Pre-Poisson submanifolds are exactly those for which $\sharp T N^{\circ}+T N$, or equivalently the image $\operatorname{Im}(\phi)$, has constant rank. 
The following table taken from [7] ${ }^{6}$ characterizes submanifolds of symplectic and Poisson manifolds in terms of $\operatorname{Im}(\phi)$ :

\begin{tabular}{l|l|l} 
& $M$ symplectic & $M$ Poisson \\
\hline $\operatorname{Im}(\phi)=0$ & $N$ coisotropic & $N$ coisotropic \\
$\operatorname{Im}(\phi)=v N$ & $N$ symplectic & $N$ cosymplectic \\
$\operatorname{Rk}(\operatorname{Im}(\phi))=$ const & $N$ presymplectic & $N$ pre-Poisson
\end{tabular}

If $\sharp T N^{\circ}$ has constant rank, then pulling back the Dirac structure $\operatorname{graph}(\pi)$ via the inclusion $N \hookrightarrow M$ one obtains a smooth Dirac structure on $N$.

When $\operatorname{char}(N)$ has constant rank and is smooth, then $\operatorname{char}(N)$ is an involutive distribution on $N$, whose quotient (when smooth) has nice properties, see $\S 4.2$.

\section{Pre-Poisson}

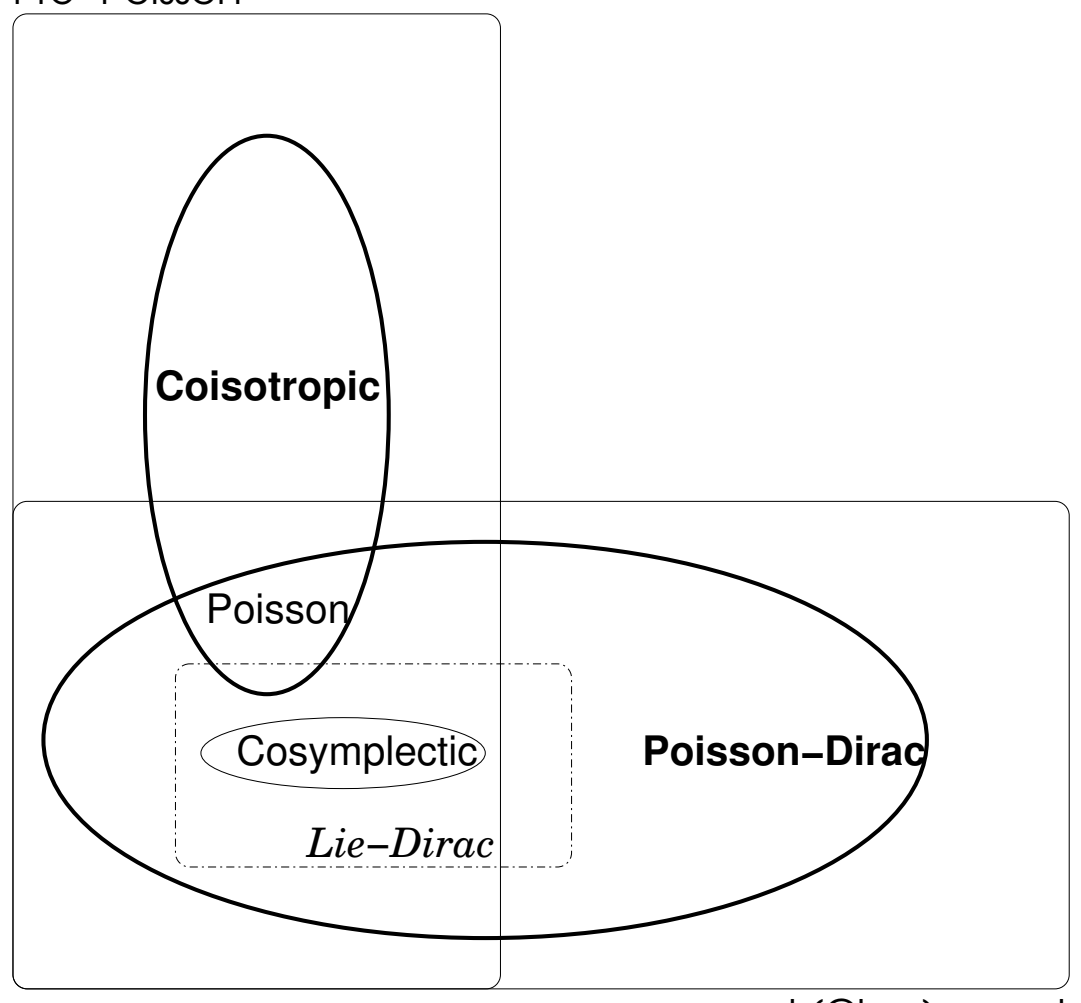

$\operatorname{rank}($ Char $)=$ const.

Fig. 1 Relation between the classes of submanifolds considered in this note. Recall that a submanifold $N$ is Pre-Poisson iff $\operatorname{rank}\left(\sharp T N^{\circ}+T N\right)=$ const . and that $\operatorname{char}(N)=\sharp T N^{\circ} \cap T N$.

${ }^{6}$ [7] considers the map $p r_{V N} \circ \sharp: T N^{\circ} \rightarrow v N$, whose image is of course the same as the one of $\phi$. 


\subsection{Embeddings of pre-Poisson submanifolds}

In this Subsection we show that pre-Poisson submanifolds of $(M, \pi)$ can be regarded as coisotropic ones (in some other Poisson manifold), and hence share many properties of coisotropic submanifolds.

Given a pre-Poisson submanifold $N$, one can find constraints (defined on some open subset $U \subset M)$ that are split into first and second class constraints [3, §2.1]. More precisely, choose constraints $\left\{\varphi^{v}\right\}$ such that $\left.d \varphi^{v}\right|_{\sharp T N^{\circ}}=0$, and complete by adding other constraints $\left\{\varphi^{A}\right\}$. The map $\sharp: T N^{\circ} \rightarrow \sharp T N^{\circ}$ maps $\sharp d \varphi^{v}$ into $T N$, so the $\varphi^{v}$ are first class constraints (i.e., $\left\{\varphi^{v}, \varphi^{\mu}\right\}$ and $\left\{\varphi^{v}, \varphi^{A}\right\}$ vanish along $U \cap N$ ). Further it maps $\operatorname{span}\left\{d \varphi^{A}\right\}$ isomorphically onto a complement $W$ of $T N \cap \sharp T N^{\circ}$ in $\sharp T N^{\circ}$, and in the basis of $W$ dual to $\left.d \varphi^{A}\right|_{W}$ the isomorphism is represented by the matrix $\left\{\varphi^{A}, \varphi^{B}\right\}$. So the $\varphi^{A}$ are second class constraints (i.e., the matrix $\left\{\varphi^{A}, \varphi^{B}\right\}$ is non-degenerate along $U \cap N)$.

The zero level set of the second class constraints $\varphi^{A}$ is a cosymplectic submanifold $\tilde{M}$ of $(M, \pi)$, see $\S 3.2$. The submanifold $U \cap N \subset \tilde{M}$ is given by the remaining constraints $\left.\varphi^{V}\right|_{\tilde{M}}$, which are first class, hence $U \cap N$ is a coisotropic submanifold of $\tilde{M}$.

The above argument is a local one. One can show that the result holds globally, with a uniqueness statement:

Proposition 1. [7, Thm 3.3 and Thm. 4.3] Let $N$ be a pre-Poisson submanifold of a Poisson manifold $(M, \pi)$. Then there exists a cosymplectic submanifold $\tilde{M}$ containing $N$ such that $N$ is coisotropic in $\tilde{M}$.

Further $\tilde{M}$ is unique up to neighborhood equivalence: if $\tilde{M}_{0}, \tilde{M}_{1}$ are cosymplectic submanifolds that contain $N$ as a coisotropic submanifold then, shrinking $\tilde{M}_{0}$ and $\tilde{M}_{1}$ to a smaller tubular neighborhood of $N$ if necessary, there is a Poisson diffeomorphism from $\tilde{M}_{0}$ to $\tilde{M}_{1}$ which is the identity on $N$.

The above proposition does not imply that all questions involving pre-Poisson submanifolds can be reduced to questions about coisotropic ones. For instance in $[2, \S 6]$ the authors consider two distinct pre-Poisson submanifolds $N_{1}$ and $N_{2}$ with non-empty intersection, and in general it is not possible to find a cosymplectic submanifold containing coisotropically both $N_{1}$ and $N_{2}$.

\subsection{Quotients of pre-Poisson submanifolds}

In this Subsection we show that every submanifold $N$ of a Poisson manifold has an associated "reduced" Poisson algebra which - when certain assumptions on $N$ are satisfied - corresponds to the quotient of $N$ by $\operatorname{char}(N)=T N \cap \sharp T N^{\circ}$. We follow $[7, \S 6]$.

For any submanifold $N$ of $(M, \pi)$, consider again the multiplicative ideal $\mathscr{I}:=$ $\left\{f \in C^{\infty}(M):\left.f\right|_{N}=0\right\}$ of the Poisson algebra $C^{\infty}(M)$. Its Poisson normalizer 


$$
\mathscr{F}:=\left\{\hat{f} \in C^{\infty}(M):\{\hat{f}, \mathscr{I}\} \subset \mathscr{I}\right\}
$$

is a Poisson subalgebra of $C^{\infty}(M)$, and by construction $\mathscr{F} \cap \mathscr{I}$ is a Poisson ideal in $\mathscr{F}$. Hence the quotient $\mathscr{F} /(\mathscr{F} \cap \mathscr{I})$ is a Poisson algebra. Notice that $\mathscr{F} /(\mathscr{F} \cap$ $\mathscr{I})$ is exactly the subset of functions $f$ on $N$ which admits an extension to some function $\hat{f} \in C^{\infty}(M)$ whose differential annihilates $\sharp T N^{\circ}$ (or equivalently $\left.X_{\hat{f}}\right|_{N} \subset$ $T N)$. In geometric terms, the induced Poisson bracket on $\mathscr{F} /(\mathscr{F} \cap \mathscr{I})$ is computed as follows:

$$
\{f, g\}=\left.\{\hat{f}, \hat{g}\}\right|_{C}=\left.X_{\hat{f}}(g)\right|_{N}
$$

for extensions $\hat{f}, \hat{g} \in \mathscr{F}$, where the second Poisson bracket is the one on $C^{\infty}(M)$.

On the other hand $\operatorname{char}(N)_{p} \subset T_{p} N$ is the kernel of the bilinear form $\imath^{*} \omega_{p}$, where $(\mathscr{O}, \omega)$ is the symplectic leaf of $M$ through $p$ and $\imath: N \cap \mathscr{O} \hookrightarrow \mathscr{O}$ the inclusion. Hence, from a geometric point of view, it is natural to consider the set of basic functions on $N$, i.e.

$$
C_{\text {bas }}^{\infty}(N)=\left\{f \in C^{\infty}(N):\left.d f\right|_{\sharp T N^{\circ} \cap T N}=0\right\} .
$$

When $\operatorname{char}(N)$ is regular and smooth and the quotient $\underline{N}$ is a smooth manifold, then $C_{\text {bas }}^{\infty}(N)$ is isomorphic to $C^{\infty}(\underline{N})$.

In general we have $\mathscr{F} /(\mathscr{F} \cap \mathscr{I}) \subset C_{\text {bas }}^{\infty}(N)$. When $N$ is a pre-Poisson submanifold one has equality [2, Thm. 3]. Hence, for pre-Poisson submanifolds, the set of basic functions has a Poisson algebra structure, and whenever the quotient $\underline{N}$ is a smooth manifold, it has an induced Poisson structure.

\subsection{Relation to subgroupoids of $\Gamma(M)$}

Generalizing the fact that Lie algebras are the infinitesimal objects associated to Lie groups, Lie algebroids (see §1) are associated to so-called Lie groupoids [12, §13]. A groupoid is a category (so in particular it consists of a set of arrows with two maps $\mathbf{s}$ and $\mathbf{t}$ to the set of objects) where every arrow is invertible. For Lie groupoids we require that the sets involved in the definition be manifolds, the maps be smooth, and $\mathbf{s}, \mathbf{t}$ surjective submersions.

Let $(M, \pi)$ be a Poisson manifold. When certain obstructions vanish [10, Thm. 2], there exists a Lie groupoid whose Lie algebroid is $T^{*} M$. There exists a unique (up to isomorphism) such Lie groupoid $\Gamma(M)$ whose s-fibers are simply connected. $\Gamma(M)$ is actually a symplectic groupoid [8], i.e. it carries a symplectic form $\Omega$ such that the graph of the multiplication (composition of arrows) in $(\Gamma(M) \times \Gamma(M) \times \Gamma(M), \Omega \times \Omega \times(-\Omega))$ is Lagrangian, and so that the target map t: $\Gamma(M) \rightarrow M$ is a Poisson map. For instance, if $(M, \omega)$ is a simply connected symplectic manifold, then $(\Gamma(M), \Omega)=(M \times M, \omega \times(-\omega))$, and the groupoid multiplication of $\Gamma(M)$ is given by $(x, y) \cdot(y, z)=(x, z)$. 
Assume that the Poisson manifold $(M, \pi)$ admits a symplectic groupoid $\Gamma(M)$. "Nice" classes of (immersed) subgroupoids of $\Gamma(M)$ are given by the subgroupoids which are coisotropic or symplectic submanifolds, or more generally presymplectic submanifolds. It is natural to ask which classes of submanifolds of $M$ are the bases (sets of objects) of "nice" subgroupoids of $\Gamma(M)$. Given a submanifold $N \subset M$, any Lie subalgebroid of $T^{*} M$ over $N$ must be contained in $\sharp^{-1} T N$ (otherwise there is no induced anchor). Further, the only subbundle of $T^{*} M$ naturally associated to the submanifold $N$ is $T N^{\circ}$. Hence we are lead to consider

- $T N^{\circ} \cap \sharp^{-1} T N \quad$ (it has constant rank iff $N$ a pre-Poisson submanifold)

- $\sharp^{-1} T N \quad$ (it has constant rank iff its annihilator $\sharp T N^{\circ}$ does).

When they have constant rank, they are automatically Lie subalgebroids of $T^{*} M[7$, Prop. 3.6]. Now we look at the corresponding subgroupoids of $\Gamma(M)$.

Considering the Lie subalgebroid $T N^{\circ} \cap \sharp^{-1} T N$ we have:

Proposition 2. [7, Prop. 7.2] Let $N$ be a pre-Poisson submanifold of $(M, \pi)$. Then the subgroupoid of $\Gamma(M)$ integrating $T N^{\circ} \cap \sharp^{-1} T N$ is an isotropic subgroupoid of $\Gamma(M)$.

The above subgroupoid is Lagrangian exactly when $N$ is coisotropic [5, §5]. (In [5] this correspondence is the main tool to show that the integration of Poisson manifolds can be derived from the one of Lie algebroids). When $N$ is cosymplectic, then the above subgroupoid is the trivial groupoid $N \rightrightarrows N$.

Next assume that $\sharp T N^{\circ}$ has constant rank and consider the Lie subalgebroid $\sharp^{-1} T N$. A subgroupoid of $\Gamma(M)$ integrating it is $\mathbf{s}^{-1}(N) \cap \mathbf{t}^{-1}(N)$.

Remark 2. We saw that the graph of $\pi$ pulls back to a smooth Dirac structure on $N$. It can be shown [7, Rem. 7.3] that $\mathbf{s}^{-1}(N) \cap \mathbf{t}^{-1}(N)$, with the restriction of the sympletic form $\Omega$ on $\Gamma(M)$, is an over-pre-symplectic groupoid inducing the same Dirac structure on $N$ [1, Ex. 6.7].

Proposition 3. [7, Prop. 7.5] Let $N$ be any submanifold of $M$. Then $\mathbf{s}^{-1}(N) \cap \mathbf{t}^{-1}(N)$ is a presymplectic ${ }^{7}$ submanifold of $\Gamma(M)$ iff $N$ is pre-Poisson and char $(N)$ has constant rank. In this case the characteristic distribution of $\mathbf{s}^{-1}(N) \cap \mathbf{t}^{-1}(N)$ has rank $2 r k(\operatorname{char}(N))+r k\left(T N^{\circ} \cap T \mathscr{O}^{\circ}\right)$, where $\mathscr{O}$ denotes the symplectic leaves of $M$ intersecting $N$.

We have the following special cases: if $N$ is coisotropic and $\sharp T N^{\circ}$ has constant rank, then $\mathbf{s}^{-1}(N) \cap \mathbf{t}^{-1}(N)$ is also coisotropic; if $N$ is cosymplectic, then $\mathbf{s}^{-1}(N) \cap \mathbf{t}^{-1}(N)$ is also cosymplectic [7, Lemma. 7.1].

When $N$ is pre-Poisson and $\operatorname{char}(N)$ has constant rank, the quotient $\underline{N}$ of $N$ by $\operatorname{char}(N)$ (when smooth) is a Poisson manifold, see $\S 4.2$. As seen in Prop. $3, \mathbf{s}^{-1}(N) \cap$

\footnotetext{
${ }^{7}$ Recall that a submanifold $S$ of the symplectic manifold $(\Gamma(M), \Omega)$ is presymplectic iff its characteristic distribution $T S \cap T S^{\Omega}$ has constant rank.
} 
$\mathbf{t}^{-1}(N)$ is a Lie subgroupoid and a presymplectic submanifold of $\Gamma(M)$. When the quotient $\mathbf{s}^{-1}(N) \cap \mathbf{t}^{-1}(N)$ by its characteristic distribution (i.e., the leaf-space) is smooth, one expects it ${ }^{8}$ to be a symplectic groupoid for $\underline{N}$.

The following example, which is the only original contribution of present note, shows that this is not the case: $\mathbf{s}^{-1}(N) \cap \mathbf{t}^{-1}(N)$ usually is not even a set-theoretic groupoid.

Example 6. Let $N$ be the trivial circle bundle over the open 2-disk $D$, but with one point removed in the fiber over $0 \in D$. We write suggestively $N=D \times \hat{S}^{1}$, where $\hat{S}^{1}{ }_{p}$ denotes the circle for all non-zero $p \in D$, while $\hat{S}^{1}{ }_{0}$ is the circle with a point deleted. Notice that $\pi_{1}(N)=\mathbb{Z}$, generated by any of the circle fibers. It is easy to see that the universal cover of $N$ is $\tilde{N}=(D \times \mathbb{R})-(\{0\} \times \mathbb{Z})$. To emphatize the fact that $\tilde{N}$ is a bundle over $D$ we write $N=D \times \hat{\mathbb{R}}$, where $\hat{\mathbb{R}}_{p}=\mathbb{R}$ for non-zero $p \in D$ and $\hat{\mathbb{R}}_{0}=\mathbb{R}-\mathbb{Z}$.

Now we bring in Poisson structures. Let $M=D \times \hat{S}^{1} \times I$, where $I$ is the open interval, and endow it with the symplectic structure $\Omega$ obtained as the product of the symplectic structure on the disk $D$ and the (restriction of) the symplectic structure on $S^{1} \times I$. The symplectic groupoid of $(M, \Omega)$ is $\Gamma(M)=\left(\tilde{M} \times_{\mathbb{Z}} \tilde{M}, \mathbf{t}^{*} \Omega-\mathbf{s}^{*} \Omega\right)$, where $\tilde{M}$ denotes the universal cover of $M$ and the action of $\pi_{1}(M)=\mathbb{Z}$ is by diagonal deck transformations.

We view $N$ as a submanifold of $M$; it is a presymplectic submanifold, and clearly $\underline{N} \cong D$. We have

$$
\mathbf{s}^{-1}(N) \cap \mathbf{t}^{-1}(N)=(D \times \hat{\mathbb{R}}) \times_{\mathbb{Z}}(D \times \hat{\mathbb{R}}) .
$$

The characteristic leaves of $\mathbf{s}^{-1}(N) \cap \mathbf{t}^{-1}(N)$ almost coincide with the fibers of the natural projection onto $D \times D$ : the characteristic leaves $\operatorname{are}^{9} F_{\left(p_{1}, p_{2}\right)}=\left\{\left[\left(p_{1}, t_{1}, p_{2}, t_{2}\right)\right]\right.$ : $\left.t_{1}, t_{2} \in \mathbb{R}\right\}$ if $\left(p_{1}, p_{2}\right) \neq(0,0) \in D \times D$ (topologically these are either cylinders or rectangles), whereas sitting over $(0,0) \in D \times D$ we have the quotient of $(\mathbb{R}-\mathbb{Z}) \times$ $(\mathbb{R}-\mathbb{Z})$ by the diagonal $\mathbb{Z}$ action, which consists of countably many leaves. Hence the leaf space is

$$
\underline{\mathbf{s}^{-1}(N) \cap \mathbf{t}^{-1}(N)}=D \hat{\times} D,
$$

where the latter denotes the non-Hausdorff manifold obtained from $D \times D$ replacing $(0,0)$ with a copy of $\mathbb{Z}$.

We ask whether the projection $p r: \mathbf{s}^{-1}(N) \cap \mathbf{t}^{-1}(N) \rightarrow \mathbf{s}^{-1}(N) \cap \mathbf{t}^{-1}(N)$ induces a groupoid structure (over $\underline{N}$ ) on the quotient. We have well-defined source and target maps for $\mathbf{s}^{-1}(N) \cap \mathbf{t}^{-1}(N)$, but the groupoid multiplication of $\mathbf{s}^{-1}(N) \cap \mathbf{t}^{-1}(N)$ does not descend to the quotient. Indeed, consider $(0, p) \in D \hat{\times} D$ where $p$ is non-zero. A preimage under $p r$ is $\left[\left(0, \mu_{1}\right),(p, \lambda)\right]$ where $\mu_{1} \in \mathbb{R}-\mathbb{Z}$ and $\lambda \in \mathbb{R}$ are arbitrary. Similarly, we consider $(p, 0) \in D \hat{\times} D$ and as a preimage we pick $\left[(p, \lambda),\left(0, \mu_{2}\right)\right]$ where again $\mu_{2} \in \mathbb{R}-\mathbb{Z}$ is arbitrary. Now multiplying these two elements of $\mathbf{s}^{-1}(N) \cap \mathbf{t}^{-1}(N)$ we obtain $\left[\left(0, \mu_{1}\right),\left(0, \mu_{2}\right)\right]$. The value of its pro-

${ }^{8}$ For $N$ a Poisson-Dirac submanifold this was already pointed out in $[10, \S 8]$.

${ }^{9}$ Square brackets denote equivalence classes under the $\mathbb{Z}$-action on $(D \times \hat{\mathbb{R}}) \times(D \times \hat{\mathbb{R}})$. 
jection under $p r$ depends on the concrete choice of $\mu_{1}$ and $\mu_{2}$. This shows that $\mathbf{s}^{-1}(N) \cap \mathbf{t}^{-1}(N)$ does not have an induced groupoid structure.

In order to obtain a groupoid as a quotient of $\mathbf{s}^{-1}(N) \cap \mathbf{t}^{-1}(N)$, one would need to identify all the countably many characteristic leaves sitting over $(0,0) \in D \times D$.

To end with, we consider a Lie-Dirac submanifold $N$. By the very definition (see $\S 3.1)$ there is a canonical embedding ${ }^{10}$ of Lie algebroids $\phi: T^{*} N \rightarrow T^{*} M$, giving rise to a subgroupoid of $\Gamma(M)$. We have

Proposition 4. [15, Thm. 3.7] If $\Gamma^{\prime} \rightrightarrows N$ is a symplectic subgroupoid of $\Gamma(M)$ then $N$ is a Lie-Dirac submanifold of $(M, \pi)$. Conversely, if $N$ is a Lie-Dirac submanifold of $(M, \pi)$, then $\phi\left(T^{*} N\right)$ integrates to a symplectic subgroupoid of $\Gamma(M)$.

Acknowledgements I thank the organizers of the conference "Complex and Differential Geometry" in Hannover for the fruitful meeting and local support. This work was partially supported by the Centro de Matemática da Universidade do Porto, financed by FCT through the programs POCTI and POSI, with Portuguese and European Community structural funds, and by the FCT program Ciencia 2007.

\section{References}

1. Bursztyn, H., Crainic, M., Weinstein, A., Zhu, C.: Integration of twisted Dirac brackets. Duke Math. J. 123(3), 549-607 (2004)

2. Calvo, I., Falceto, F.: Poisson reduction and branes in Poisson-sigma models. Lett. Math. Phys. 70(3), 231-247 (2004)

3. Calvo, I., Falceto, F.: Star products and branes in Poisson-Sigma models. Commun. Math. Phys. 268(3), 607-620 (2006)

4. Calvo, I., Falceto, F., Zambon, M.: Reduction of Dirac structures along isotropic subbundles, ArXiv math.DG/0702025

5. Cattaneo, A.S.: On the integration of Poisson manifolds, Lie algebroids, and coisotropic submanifolds. Lett. Math. Phys. 67(1), 33-48 (2004)

6. Cattaneo, A.S., Zambon, M.: Pre-poisson submanifolds. In: Travaux mathématiques., Trav. Math., XVII, pp. 61-74. Univ. Luxemb., Luxembourg (2007)

7. Cattaneo, A.S., Zambon, M.: Coisotropic embeddings in Poisson manifolds. Trans. Amer. Math. Soc. 361(7), 3721-3746 (2009).

8. Coste, A., Dazord, P., Weinstein, A.: Groupoïdes symplectiques. In: Publications du Département de Mathématiques. Nouvelle Série. A, Vol. 2, Publ. Dép. Math. Nouvelle Sér. A, vol. 87, pp. i-ii, 1-62. Univ. Claude-Bernard, Lyon (1987)

9. Courant, T.J.: Dirac manifolds. Trans. Amer. Math. Soc. 319(2), 631-661 (1990)

10. Crainic, M., Fernandes, R.L.: Integrability of Poisson brackets. J. Differential Geom. 66(1), 71-137 (2004)

11. Mackenzie, K.C.H.: General theory of Lie groupoids and Lie algebroids, London Mathematical Society Lecture Note Series, vol. 213. Cambridge University Press, Cambridge (2005)

12. Cannas da Silva, A., Weinstein, A.: Geometric models for noncommutative algebras, Berkeley Mathematics Lecture Notes, vol. 10. American Mathematical Society, Providence, RI (1999)

13. Vaisman, I.: Dirac submanifolds of Jacobi manifolds, The breadth of symplectic and Poisson geometry, Progr. Math. Vol. 232, 603-622 (2005).

${ }^{10}$ The image of the embedding sits inside $\sharp^{-1}(T N)$. 
14. Weinstein, A.: The local structure of Poisson manifolds. J. Differential Geom. 18(3), 523-557 (1983)

15. Xu, P.: Dirac submanifolds and Poisson involutions. Ann. Sci. École Norm. Sup. (4) 36(3), 403-430 (2003) 